\title{
Role of nutritional programming in determining adult morbidity
}

The idea that nutrition during critical windows in early life could influence or 'program' long term development and major disease in adulthood has important biological and public health implications. But is this concept biologically plausible? How good is the current evidence in man and should it influence practice?

\section{The concept of 'programming'}

Generally events in early life might influence long term outcome in three ways ${ }^{1}$ : (i) direct damage (for example, loss of a limb due to vascular accident); (ii) induction, deletion, or impaired development of a somatic structure resulting from a stimulus or insult during a critical period; or (iii) physiological 'setting' by an early stimulus or insult at a critical period, with long term consequences for function. The term 'programming' has been applied to the latter two processes in which the programming stimulus only exerts long term effects when applied at a 'critical' or 'sensitive' period. ${ }^{1}$

Evidence for programming, other than by nutrition, is considerable. Illustrative examples are cited here. ${ }^{1}$ Early 'imprinting' of behaviour in birds has been recognised for centuries. $^{2}$ Hormonal signals operating during critical windows have numerous programming effects. Thus, testosterone secreted locally by the fetal testis at a critical period programs development of male internal genitalia. In rats a single dose of testosterone given at the right moment to a female fetus will permanently reorientate sexual behaviour. $^{3}$ Teratogenic drugs, recognised since the 1920 s, have powerful programming effects on somatic development. A single dose of phenobarbitone given to a neonatal rat may induce lifelong change in the activity ${ }^{2}$ of a key enzyme, cytochrome P-450 dependent monooxygenase. ${ }^{4}$ Normal visual inputs are essential for development of the visual pathway, hence squint amblyopia. Programming events are common in fetal development, but for some programming stimuli the sensitive period is postnatal, and arguably, in the case of antigen induced programming of the immune system, sensitivity may be life long.

These examples indicate that programming in fetal or postnatal life may result in: initiation of normal development processes (resulting from endogenous signalling); a lasting adaptation to an early environmental stimulus; or an adverse response to an insult at a sensitive period. Theoretically, nutritional programming might operate in any of these ways.

\section{Nutritional programming in animals}

Nutritional programming has been demonstrated convincingly in animals. Manipulation of fetal and neonatal nutrition has lifelong effects on many systems. Extensive animal data, largely on rats, show nutrition at a vulnerable period of brain development may have permanent effects on brain size, brain cell number, behaviour, learning, and memory. ${ }^{56}$ In Smart's review of 165 animal studies on early undernutrition and later learning ${ }^{6}$ the number of studies in which undernourished animals fared worse than controls greatly outweighed those which favoured the controls. The extent to which these animal data have relevance to human cognitive development, however, is uncertain.

With regard to health outcomes, experimental studies on fetal nutrition have shown, for instance, that protein undernourished fetuses had long term reduction in pancreatic cells and insulin secretion ${ }^{7}$ and higher blood pressure. ${ }^{8}$ Hahn manipulated litter size in neonatal rats so that rats from small litters were temporarily overfed during the brief suckling period and found that in adulthood these animals had a permanent increase in plasma insulin and cholesterol. ${ }^{9}$ Weaning these animals on to a high carbohydrate diet further induced a lifelong increase in the activities of 3-hydroxy-3-methylglutaryl coenzyme A reductase and fatty acid synthetase (key enzymes for cholesterol and fat synthesis).

In primates (baboons) overfeeding during infancy in the female resulted in obesity that did not manifest until early adult life, ${ }^{10}$ raising the question of where the 'memory' of the early event had been stored in the intervening period. In further studies, baboons were randomly assigned to breast feeding or formula ${ }^{11}$ and then both groups placed on a 'western-style' high saturated fat diet. The previously breast fed group had, in adult life, higher plasma low density lipoprotein (LDL) and very low density lipoprotein cholesterol, lower 'protective' high density lipoprotein (HDL) cholesterol and increased cholesterol absorption perhaps relating to the permanent change in bile acid secretion. These data imply that breast feeding may program these primates to be conservative with cholesterol but that this might be disadvantageous to lipid metabolism if they were subsequently placed, unphysiologically, on a high saturated fat diet. Indeed, at postmortem examination the previously breast fed baboons had significantly more artherosclerosis than those fed formula.

Collectively, the animal data show that brief periods of early dietary manipulation have lifelong effects on neurodevelopmental and health outcomes that would be of considerable public health significance if they applied to humans.

\section{Nutritional programming in humans}

Given the evidence for programming in general and the evidence for nutritional programming in animals, nutritional programming in humans might be predicted. This has not been easy to prove, largely because most studies have not had an experimental design but have documented retrospective epidemiological associations often subject to alternative explanation. Collectively, the human data are extensive and illustrative studies are discussed here.

\section{Early nutrition and long term neurodevelopment in} humans

Considerable effort has been invested in testing the hypothesis, supported by animal data (above), that suboptimal nutrition at a vulnerable stage in brain development has permanent effects on cognitive function. Epidemiological associations found between malnutrition and reduced cognitive performance, however, might 
not be causal. ${ }^{12}$ Malnutrition, principally studied in developing countries, is inextricably associated with poverty, poor social circumstances, and lack of stimulation which might explain the adverse outcomes. Prospective, randomised, or satisfactorily controlled studies are rare and most do not provide unequivocal data.

Recently more formal studies have provided stronger evidence that nutrition could influence cognitive development. ${ }^{13}$ A study of premature babies strictly randomised to a preterm versus standard formula for only one month showed, at blind follow up, major advantages in neurodevelopment at 18 months $^{14}$ and in verbal IQ at 7.5-8 years (A Lucas, unpublished). Whether this sensitivity to early nutrition applies to infants born at term remains a major area for study, with implications for infants with undernutrition complicating many clinical disease states.

Several studies suggest breast feeding promotes long term neurodevelopment, in some cases even after attempts to adjust for confounding factors, ${ }^{15}$ though whether or not these effects reflect residual confounding by educational and parenting differences between groups is uncertain. The evidence that human milk may promote neurodevelopment and IQ in infants born preterm is stronger, ${ }^{1516}$ with implications for clinical management. Such effects would be biologically plausible as human milk contains factors including hormones and long chain polyenoic fatty acids (currently under intensive investigation ${ }^{17}$ ) that could theoretically influence neurodevelopment.

Some specific nutrients have been claimed to be important for brain development. A large body of data links iron deficiency with reduced cognitive function and possibly a permanent reduction. ${ }^{18}$ These data are supported by animal studies showing long term effects of iron deficiency on the brain, notably reduction in dopamine receptors. ${ }^{19}$ However, as in malnutrition studies, iron deficiency anaemia is linked with poor social circumstances, confounding outcome studies. While current evidence renders it unwise to allow infants to develop iron deficiency, proof that it adversely programs neurodevelopment is awaited.

\section{Nutrition and later disease}

Most studies on early nutrition and later health have been epidemiological and inconclusive. Despite considerable interest in lipid intake in early life no convincing association with later atherosclerosis has yet emerged. Unlike the studies in baboons (above), breast and bottle fed infants have not been shown to differ in later total plasma cholesterol at 8 years $^{20}$ or total cholesterol, LDL, or HDL at up to 16 years (unpublished). Early salt intake has been associated with later high blood pressure in some studies but randomised trials have failed to show a causal link in either normal or preterm individuals. ${ }^{21}$

Breast and formula fed babies may have potentially important differences in later health outcomes, though these might be confounded by the major demographic differences between groups. For instance, breast feeding has been associated with a protective effect against insulin dependent diabetes ${ }^{22}$ (not observed by all) and a reduced incidence of lymphoma. ${ }^{23}$ Whether these associations are causal needs further exploration.

To help resolve the uncertainty over the effects of early nutrition on long term health, a series of long term prospective and strictly randomised dietary studies were started in 1982 by Lucas and coworkers. ${ }^{24}$ Initial studies have been in preterm infants. Follow up to 7.5-8 years shows that a brief period of early dietary management had a major impact on later neurodevelopment and IQ (above), pattern of allergic reactions and atopy, waist-hip ratio, linear growth, and bone mineralisation. That such a brief period of dietary manipulation (four weeks on average) had such significant, and in the case if cognitive development, probably permanent effects, underlies the sensitivity to nutritional programming during the perinatal period in humans. Cardiovascular risk and bone outcomes are currently being explored in these children and in others born at term to further test formally the hypothesis that early nutrition programs propensity to adult degenerative disease.

\section{Size in early life and later morbidity}

In the past seven years a series of studies, notably those by Barker, Hales and coworkers, have shown relationships between anthropometric indices at birth and at 1 year and cardiovascular disease and its risk factors. ${ }^{25} 26$ Low body weight, head circumference, and ponderal index at birth and low weight at 1 year have been associated with increased risk of later cardiovascular disease. Small size at birth and up to 1 year has also been associated with higher blood pressure and adverse changes in plasma concentrations of glucose, insulin, fibrinogen, factor VII, and apolipoprotein $\mathrm{B}$; abdominal circumference at birth is inversely associated with raised serum concentration of total cholesterol, LDL cholesterol, and apolipoprotein B. These provoking and important observations have been interpreted by the investigators as supporting the hypothesis that poor fetal nutrition, perhaps resulting from poor maternal nutrition, adversely programmes the individual for later cardiovascular disease, hypertension, and diabetes. Their suggestion is that improvement of fetal nutrition might be an important public health measure. ${ }^{26}$

In favour of this thesis is the evidence that early nutrition in animals has been shown to program corresponding outcomes. There are inconsistencies, however: in rats, overnutrition rather than undernutrition may be associated with later increase of blood cholesterol ${ }^{9}$ and chronic undernutrition has been associated with longevity. ${ }^{27}$ The relationship between low birth weight and a later adverse outcome might be explained by confounding demographic factors, though attempts to account for known potential confounders has not displaced the observed associations. An area for debate, however, is the proposed nutritional interpretation. Poor intrauterine growth might be associated with other, non-nutritional derangements that could be responsible for long term programming. In order to extricate the effects of nutrition from other possible intrauterine factors that might influence later blood pressure, a study of 758 babies born preterm was undertaken. ${ }^{28}$ These babies were strictly randomly assigned to diet before the equivalent of full term. Even extremes of nutritional intake and of growth performance had no effect on blood pressure at 8 years. These data indicate that for blood pressure, at least, poor nutrition and growth per se may not be the explanation for the relationship between low birth weight and later blood pressure. It has also been counterargued that poor maternal nutrition is not a critical factor for fetal growth retardation in the West, ${ }^{29}$ suggesting attempts to improve fetal nutrition via maternal nutrition might prove ineffective.

Size at birth has emerged as a significant risk marker for later health. There remains, however, the need for further work to explore whether nutritional programming is the explanation in order to underpin public health practice. 


\section{How does programming work?}

Nutritional programming has been convincingly demonstrated in animals including primates and there is now compelling evidence from experimental studies that this process operates in humans. More recently, attention has turned to mechanism. ${ }^{1}$ Some programming events might have immediate effects on structural development, for example, on dendritic arborisation or glial cell growth in the brain, with long term consequences. However, nutritional programming here might not simply reflect failure to fuel a growth process. Nutrients might be critical signals acting directly or via coupling mechanisms on 'receptors' in sensitive tissues. With regard to the programming or 'setting' of later function, for instance of a key metabolic pathway, the question is how the 'memory' of an early event is 'stored' throughout life despite continuous cellular replication and replacement. Proposed mechanisms include adaptive effects on gene expression transmitted to the progeny of the originally programmed cells. ${ }^{1}$ Alternatively, the early nutritional milieu may stimulate cellular proliferation or adaptive clonal selection so that the quantity or proportion of cell populations in a tissue are permanently affected. Evidence in support of the latter thesis is the recent animal work showing that prenatal protein restriction results in lifelong differential expression of enzymes located in periportal and perivenous hepatic cell populations, ${ }^{30}$ suggesting that the early programming event may have been a stimulus to differential growth of clones of early hepatocytes. Arguing teleologically, it would make good biological sense for the organism to use such cell biological strategies to fine tune its metabolic machinery adaptively after sensing the early environmental milieu.

\section{Clinical implications and future directions}

Medical and public health practice is ideally underpinned by research that establishes outcome benefit. The perceived benefits from infant nutritional practices have traditionally included the promotion of short term growth to accepted reference rates and of nutrient retention sufficient to fuel estimated requirements and prevent insufficiencies. The possibility that nutrition in early life may have major effects on later health and development totally changes the targets for infant nutritional research. Indeed, with the exception of the early nutritional care of preterm infants which can now be considered in relation to long term outcome data, for normal infants, let alone fetuses, there is hardly a nutritional management policy or recommended dietary allowance that could be adequately scientifically defended in terms of its beneficial effects in later life.

Can we then readjust our early nutritional policies on the basis of new observations relating to nutritional programming? Unfortunately, most published data are not based on outcome studies and cannot readily be translated into management policy. For instance, should we feed small infants intensively to make them large by 1 year, or is intensive feeding of a baby set up prenatally to be small the worst programming stimulus? It is disquieting that some current officially recommended feeding policies might turn out not to promote optimal future health.

Fortunately, the intriguing intellectual problem of unravelling nutritional programming is drawing into the infant feeding arena the largest wave of research talent, from basic scientists to adult physicians, that the field has ever seen. Hopefully, our children's children will benefit.

ALAN LUCAS

Dunn Nutritional Laboratory,

Downhams Lane,

Milton Road

Cambridge CB4 $1 X \mathfrak{F}$

This annotation is dedicated to Professor Dame June Lloyd.

1 Lucas A. Programming by early nutrition in man. In: Bock GR, Whelan J, eds. The childhood environment and adult disease. (CIBA Foundation Symposium 156.) Chichester: Wiley, 1991: 38-55.

2 Smart JL. Critical periods in brain development. In: Bock GR, Whelan J, eds. The childhood environment and adult disease. (CIBA Foundation Symposium 156.) Chichester: Wiley, 1991: 109-28.

3 Angelbeck JH, Du Brul EF. The effect of neonatal testosterone on specific male and female patterns of phosphorylated cytosolic proteins in the rat preoptic-hypothalamus, cortex and amygdala. Brain Res 1983; 264: 277-83.

4 Bagley DM, Hayes JR. Neonatal phenobarbital administration results in increased cytochrome P450-dependent monoocygencase activity in adult increased cytochrome P450-dependent monoocygencase activity in aduit

5 Dobbing J. Nutritional growth restriction and the nervous system. In: Davison AN, Thompson RHS, eds. The molecular bases of neuropathology. London: Edward Arnold, 1981: 221-33.

6 Smart J. Undernutrition, learning and memory: review of experimental studies. In: Taylor TG, Jenkins NK, eds. Proceedings of XII international congress of nutrition. London: John Libbey, 1986: 74-8.

7 Snoek A, Remacle C, Reusens B, Hoet JJ. Effect of a low protein diet during pregnancy on the fetal rat endocrine pancreas. Biol Neonate 1990; 57: pregnan.

8 Barker DJP, Gluckman PD, Godfrey KM, Harding JE, Owens JA, Robinson JS. Fetal nutrition and cardiovascular disease in adult life. Arch Dis Child 1993; 341: 938-41.

9 Hahn P. Effect of litter size on plasma cholesterol and insulin and some liver and adipose tissue enzymes in adult rodents. $\mathcal{f}$ Nutr 1984; 114: 1231-4.

10 Lewis DS, Bartrand HA, McMahan CA, McGill HC Jr, Carey KD, Masoro EJ. Preweaning food intake influences the adiposity of young adult baboons. $₹$ Clin Invest 1986; 78: 899-905.

11 Mott GE, Lewis DS, McGill HC. Programming of cholesterol metabolism by breast or formula feeding. In: Bock GR, Whelan J, eds. The childhood environment and adult disease. (CIBA Foundation Symposium 156.) Chichester: Wiley, 1991: 56-76.

12 Grantham-McGregor S. Field studies in early nutrition and later achievement. In: Dobbing J, ed. Early nutrition and later achievement. London: Academic Press, 1987: 128-74.

13 Smart JL. Behavioural consequences of undernutrition. Proc Nutr Soc 1993; 52: 189-99.

14 Lucas A, Morley R, Cole TJ, et al. Early diet in preterm babies and developmental status at 18 months. Lancet 1990; 335: 1477-81.

15 Lucas A, Morley RM, Cole TJ, Lister G, Leeson-Payne C. Breast milk and subsequent intelligence quotient in children born preterm. Lancet 1992; 339: 261-4.

16 Lucas A, Morley RM, Cole TJ, Gore SM. A randomised multicentre study of human milk versus formula and later development in preterm infants. Arch Dis Child 1994; 70: F141-6.

17 Innis SM. Essential fatty acids in growth and development. Prog Lipid Res 1991; 30: 39-103.

18 Lozoff B, Jimenez E, Wolf AW. Long term developmental outcome of infants with iron deficiency. $N$ Engl F Med 1992; 325: 687-94.

19 Youdim MBH. Neuro-pharmacological and neuro-biochemical aspects of iron deficiency. In: Dobbing J, ed. Brain behaviour and iron in the infant diet. London: Springer Verlag, 1990: 83-105.

20 Fomon ST, Rogers RR, Zeigler EE, Nelson SE, Thomas LN. Indices of fatness and cholesterol at age eight years in relation to feeding and growth in early infancy. Pediatr Res $1984 ; 18: 1233-8$.

21 Lucas A, Morley R, Hudson GJ, et al. Early sodium intake and later blood pressure in preterm infants. Arch Dis Child 1988; 63: 656-7.

22 Klingensmith GJ. Reduced risk of IDDM among breast-fed children Diabetes 1988; 37: 1625-32.

23 Davis MK, Savitz DA, Graubard BI. Infant feeding and childhood cancer. Lancet 1988; ii: 365-8.

24 Lucas A, Gore SM, Cole TJ, et al. A multicentre trial on feeding low birthweight infants: effects of diet on early growth. Arch Dis Child 1984; 59: 722-30.

25 Fall CHD, Barker DJP, Osmond C, Winter PD, Clark PMS, Hales CN Relation of infant feeding to adult serum cholesterol concentration and death from ischaemic heart disease. In: Barker DJP, ed. Fetal and infant origins of adult disease. London: $\mathrm{BMJ}$, 1992: 275-88.

26 Barker DJP, Martyn CN, Osmond C, Hales CN. Growth in utero and

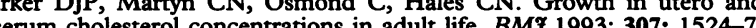
.

27 McCay CM, Maynard L, Sperling G, Barnes LL. Retarded growth, life span, ultimate body size, and age changes in
diets restricted in calories. $₹$ Nutr $1939 ; 18: 1$.

28 Lucas A, Morley $R$. Does early nutrition program later blood pressure? $B M \mathcal{F}$ 1994; 309: 304-8.

Walker ARP, Walker BF. Fetal nutrition and cardiovascular disease in adult life. Lancet 1993; 341: 1421 .

30 Desai M, Crowther N, Lucas A, Hales CN. Programming of hepatic metabolism by low protein diet during early life. British Diabetic Association Medical and Scientific Section Spring Meeting, Bournemouth 1994. 